\title{
Preparo do of talmologista para o tratamento das reações adversas na retinografia fluoresceínica
}

\author{
Evaluation of knowledge of theophthalmologists regarding for the treatment of adverse \\ reactions in fluorescein angiography
}

\author{
Rodrigo Pessoa Cavalcanti Lira ${ }^{1}$ \\ Alessandra Pereira Dantas ${ }^{2}$ \\ Luiz Antônio Trigueiro ${ }^{3}$ \\ Patrícia Fernanda Suassuna de Farias ${ }^{4}$
}

\begin{tabular}{|c|}
\hline RESUMO \\
\hline $\begin{array}{l}\text { Objetivos: Avaliara estrutura das clínicas que realizam retinografia fluores- } \\
\text { ceínica em Pernambuco e, por meio de questionário, avaliar o preparo do } \\
\text { oftalmologista para contornar reações adversas relativas ao exame e deter- } \\
\text { minar se o tempo de conclusão do curso de especialização influencia nos } \\
\text { resultados. Métodos: Foi realizada entrevista com os } 18 \text { médicos nas dez } \\
\text { clínicas que realizam este exame. O questionário era de múltipla escolha com } \\
\text { dez questões. Versava sobre aspectos das reações adversas. Resultados: } \\
\text { Um médico recusou-se a participar e foi excluído. Entre os demais, quinze } \\
\text { ( } 88,2 \% \text { ) possuíam o título de especialista. Todas as clínicas estavam bem } \\
\text { aparelhadas em relação à estrutura básica de equipamentos e medicações } \\
\text { para o atendimento inicial de reações adversas. Metade das clínicas (cinco) } \\
\text { realizava o exame com a presença de um anestesista na sala e a outra metade } \\
\text { dispunha de um anestesista de sobreaviso dentro da instituição. O número } \\
\text { de acertos obtidos pelos médicos variou de } 3 \text { a } 8 \text {, com média de } 5,2 \pm 1,6 \\
\text { acertos. Apenas quatro ( } 23,6 \% \text { médicos obtiveram nota igual ou superior } \\
\text { a sete acertos. Médicos com até cinco anos de conclusão do curso de } \\
\text { especialização obtiveram melhor desempenho no questionário (p<0,001). } \\
\text { Conclusões: Apesar das clínicas avaliadas contarem com boa estrutura } \\
\text { física e disponibilidade de assistência por anestesista, os resultados deste } \\
\text { estudo sugerem que a formação dos médicos oftalmologistas que realizam } \\
\text { o exame de retinografia fluoresceínica apresenta deficiências em relação à } \\
\text { condução de reações adversas deste contraste injetável, principalmente em } \\
\text { médicos com mais de cinco anos de conclusão do curso de especialização. }\end{array}$ \\
\hline
\end{tabular}

Descritores: Angiofluoresceinografia, Fluoresceína/efeitos adversos; Meios de contraste; Náusea/induzido quimicamente; Vômito/induzido quimicamente; Anafilaxia; Especialidade médica/educação; Questionários

\section{INTRODUÇÃO}

A angiografia retiniana com fluoresceína é uma técnica usada na interpretação de patologias oculares. Ela permite a visualização seqüencial do fluxo sangüíneo simultaneamente na retina, coróide e íris, e fornece suporte diagnóstico a impressões clínicas baseado nas alterações da dinâmica fluídica resultante de processos patológicos oculares. Embora este exame já venha sendo largamente utilizado há mais de 40 anos, o seu emprego na prática clínica ainda encontra-se em expansão graças à crescente sofisticação dos aparelhos, e a melhora da capacidade de interpretação dos resultados ${ }^{(1)}$.

A maioria das reações adversas relativas a este exame é leve. As mais freqüentes são náusea e vômitos, as quais ocorrem em aproximadamente $5 \%$ dos pacientes. Porém há vários relatos de reações graves como edema 
de glote, broncoespasmo, choque circulatório, e, inclusive, óbito (um caso em 220.000 exames) ${ }^{(2-6)}$. Embora os eventos adversos que ameaçam a vida sejam infreqüentes, é fundamental que os médicos e clínicas que realizam o exame estejam aptos para manejar adequadamente estas situações.

Os objetivos deste breve estudo foram avaliar a estrutura física e logística das clínicas que realizam retinografia fluoresceínica em Pernambuco e, através de um curto questionário de múltipla escolha, avaliar o preparo do médico oftalmologista para contornar reações adversas relativas ao exame e determinar se o tempo de conclusão do curso de especialização em oftalmologia influencia nos resultados.

\section{MÉTODOS}

No estado de Pernambuco, Brasil, atuam 267 oftalmologis$\operatorname{tas}^{(7)}$, havendo 10 clínicas e hospitais oftalmológicos que realizam o exame de retinografia fluoresceínica, sendo 8 na capital, Recife, e 2 no interior, em Caruaru e Petrolina. Dezoito oftalmologistas realizam rotineiramente esse exame nestes estabelecimentos.

Por meio de entrevista pessoal, realizada por um médico treinado, foi preenchida uma ficha de coleta de dados contendo as seguintes informações: 1) se o médico realizador do exame possuía o título de especialista emitido pelo Conselho Brasileiro de Oftalmologia (CBO); 2) se a clínica estava aparelhada com o material para atendimento inicial em caso de ocorrência de reações adversas à injeção do contraste fluoresceína, incluindo aparelhos e medicações (oxigênio, estetoscópio, esfignomanômetro, tubos endotraqueais, laringoscópios e substâncias como metoclopramida, anti-histamínicos, adrenalina, corticosteróide, aminofilina, nifedipina, diuréticos, glicose hipertônica) $\left.{ }^{(1,8-9)} ; 3\right)$ se o exame era realizado com a supervisão de anestesista ou clínico para assistência médica complementar nas reações adversas; 4) um questionário objetivo de múltipla escolha com 10 (dez) questões (em anexo), apresentando cinco alternativas e apenas uma resposta considerada correta; e 5) tempo de conclusão do curso de especialização em oftalmologia. Este questionário versava sobre aspectos das reações adversas em si, e deveria ser respondido pelo médico entrevistado, individualmente, durante a entrevista, num prazo determinado de 15 minutos. Foi dada uma nota de 0 a 10 pontos de acordo com a proporção de respostas certas. Foi determinada a nota igual ou superior a 7 (sete) pontos como satisfatória. O questionário foi testado previamente, tendo sido aplicado a três médicos clínicos experientes os quais acertaram em média $90 \%$ das questões e opinaram que, em relação ao nível de dificuldade, foram incluídas seis questões consideradas mais fáceis (perguntas 1, 2, 5, 8, 9 e 10) e quatro questões consideradas mais difíceis (perguntas 3, 4, 6 e 7).

O questionário foi elaborado por dois anestesistas com vasta experiência em anestesia e em tratamento de pacientes em unidades de terapia intensiva (UTI).

Os médicos foram divididos em dois grupos em relação ao tempo de conclusão do curso de especialização em oftalmolo- gia: o primeiro grupo composto por médicos com até 5 anos de conclusão e o segundo composto por médicos com mais de 5 anos de conclusão.

Foram feitos testes para detectar diferenças entre variáveis, usando a análise de variância (one-way ANOVA) para variáveis quantitativas. Como parâmetro de tendência central e dispersão para os dados foram determinadas, a média e o desvio padrão. Os resultados desta análise foram considerados significantes se $p<0,05$. A análise estatística foi executada com o auxílio do software EpiInfo ${ }^{\mathrm{TM}} 2000$ (Centers for Disease Control and Prevention, Atlanta, Georgia, USA).

\section{RESULTADOS}

Um médico recusou-se a participar por motivos pessoais e foi excluído do estudo. Entre os demais, quinze médicos $(88,2 \%)$ tinham título de especialista do CBO e dois $(11,8 \%)$ não o possuíam. Todas as clínicas estavam bem aparelhadas em relação à estrutura básica de equipamentos e medicações para o atendimento inicial de reações adversas ao contraste. Metade das clínicas (cinco) realizava o exame com a presença de um anestesista na sala e a outra metade dispunha de um anestesista de sobreaviso dentro da instituição, mas fora da sala de exame.

O número de acertos obtidos pelos médicos variou de 3 a 8 , com média de 5,2 $\pm 1,6$ acertos. Quatro $(23,6 \%)$ médicos obtiveram nota igual ou superior a sete acertos e $13(76,4 \%)$ médicos tiveram nota inferior a sete acertos. A média das notas obtida pelos 15 médicos com título do CBO foi de 5,3 $\pm 1,6$ acertos e a média alcançada pelos dois médicos sem o título do $\mathrm{CBO}$ foi de $4,0 \pm 1,4$ acertos $(\mathrm{p}<0,279)$.

Ao se considerar dois grupos distintos em relação ao tempo de conclusão do curso de especialização em oftalmologia, o primeiro grupo, com até 5 anos de conclusão, foi composto de 5 médicos (com média de 2,2 $\pm 1,3$ anos) e o segundo grupo, com mais de 5 anos de conclusão, foi composto de 12 médicos (com

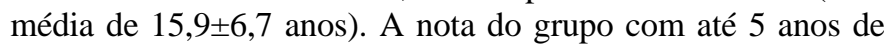
conclusão do curso foi de $7,0 \pm 1,2$ e a do grupo com mais de 5 anos de conclusão foi de $4,4 \pm 1,0$ ( $p<0,001)$.

\section{DISCUSSÃo}

O breve questionário proposto neste estudo procurou abordar alguns dos principais aspectos das reações adversas à injeção de contraste. Foram incluídos temas relacionados ao tratamento de náuseas e vômitos, profilaxia, fisiopatologia, diagnóstico e tratamento de reações anafiláticas e/ou anafilactóides, além do diagnóstico e tratamento de parada cardiorespiratória. Na condição de instrumento de avaliação subjetiva, é passível de críticas, entretanto foge da ambição deste estudo esgotar o tema ou determinar um questionário definitivo e/ou extenso sobre o assunto. Como esperado, de uma maneira global, os índices de acerto foram maiores nas questões previamente consideradas mais fáceis.

Considerando que as 10 questões envolveram tópicos fre- 
Questionário sobre o preparo do oftalmologista para o manejo das reações adversas em retinografia fluoresceínica, Pernambuco, 2004

1 - Qual destas drogas pode, mais freqüentemente, desencadear uma síndrome extrapiramidal quando usada para prevenção e/ou tratamento de náuseas e vômitos?
a) Droperidol
b) Bromoprida
c) Dramim B6
d) Metoclorpramida
e) Ondasedron

2 - Um paciente que irá submeter-se a angiofluoresceinografia, relata que tem alergia a iodo e a crustáceos. A conduta mais adequada é:

a) Administração de atropina, cimetidina e corticóide

b) Dessensibilização com contraste cutâneo

c) Administração de corticóide e difenidramina

d) Administração de butirofenonas, atropina e corticóide

e) Suspender o exame, pois o paciente tem contra-indicação absoluta

3 - Qual a manifestação clínica mais comum na Anafilaxia?

a) Taquicardia

b) Hipotensão

c) Alterações cutâneas

d) Náuseas e vômitos

e) Broncoespasmo

4 - Qual é o mais importante mediador químico e a única substância essencial para que a anafilaxia ocorra?

a) Linfocinas

b) Enzimas neutrofílicas

c) Cininas

d) Histamina

e) SRS-A

5 - Qual é o mediador químico responsável pela importante broncoconstricção, muitas vezes de difícil tratamento, nas reações anafilactóides?
a) Cininas
b) Histaminas
c) SRS-A
d) Leucotrienos
e) Enzimas neutrofílicas

6 - Fazem parte do tratamento imediato das reações anafilactóides, exceto:
a) Aminofilina
b) Adrenalina
c) Ventilação com 0 a $100 \%$
d) Interromper a administração da droga
e) Expansão da volemia

7 - As doses da adrenalina e da difenidramina endovenosa (antihistamínico venoso) nas reações anafilactóides são, respectivamente:
a) $1 \mu \mathrm{g} / \mathrm{kg}-10 \mathrm{mg} / \mathrm{kg}$
b) $5 \mu \mathrm{g} / \mathrm{kg}-0,5$ a $1 \mathrm{mg} / \mathrm{kg}$
c) $10 \mathrm{mg} / \mathrm{kg}-5 \mathrm{mcg} / \mathrm{kg}$
d) 0,1 a $0,5 \mathrm{mg} / \mathrm{kg}-5 \mathrm{mg} / \mathrm{kg}$
e) 0,5 a $1 \mathrm{mg} / \mathrm{kg}-5 \mathrm{mcg} / \mathrm{kg}$

8 - A importância e a dose dos corticoesteróides endovenoso nas reações anafilactóides são, respectivamente:

a) Agir na fase aguda - Dexametasona $4 \mathrm{mg}$ ou Hidrocortisona 0,5 a $1 \mathrm{~g}$ b) Agir na fase tardia - Dexametasona $4 \mathrm{mg}$ ou Hidrocortisona $0,5 \mathrm{a} 1 \mathrm{~g}$

c) Agir na fase tardia - Dexametasona $0,4 \mathrm{mg}$ ou Hidrocortisona 0,5 a $1 \mathrm{mg}$

d) Agir na fase aguda e tardia - Dexametasona $4 \mathrm{mg}$ ou Hidrocortisona 0,5 a $1 \mathrm{~g}$

e) Agir na fase tardia - Dexametasona 0,4mg ou Hidrocortisona 0,5 a $1 \mathrm{~g}$

9 - O diagnóstico de parada cardiorespiratória é determinado por:

a) Inconsciência

b) Inexistência de movimentos respiratórios

c) Midríase paralítica

d) Ausência de pulso na circulação central

e) Cianose

10 - Na reanimação cardiorespiratória na presença de um reanimador e de dois reanimadores qual deverá ser a relação massagemventilação geralmente preconizada:
a) $15: 2-5: 1$
b) $5: 1-2: 1$
c) $10: 2-4: 2$
d) $15: 2-10: 1$
e) $10: 4-5: 1$

Respostas ao questionário consideradas corretas: 1-D; 2-C; 3-C; 4-D; 5-C; 6-A; 7-B; 8-B; 9-D; 10-A

qüentemente abordados no ensino da graduação em medicina, é preocupante a média de acertos de apenas $5,2 \pm 1,6$, principalmente levando-se em consideração que apenas quatro $(23,6 \%)$ médicos obtiveram nota igual ou superior a sete. A média das notas obtida pelos médicos com título de especialista do CBO foi superior à média alcançada pelos médicos sem este título, entretanto a amostra foi insuficiente para demonstrar uma diferença estatisticamente significativa, mas mesmo considerando apenas os com título do CBO a média foi de apenas 5,3 $\pm 1,6$ acertos.

Um dado que chama atenção neste estudo é que os médicos com até cinco anos de conclusão do curso de especialização apresentaram um desempenho no questionário bastante superior aos médicos com mais de cinco anos de conclusão da referida etapa acadêmica $(p<0,001)$. Discussões sobre programas de educação médica continuada, no Brasil e nos Estados Unidos, têm ocupado cada vez mais espaço, na última década, não só por parte do governo como nas instituições não governamentais relevantes como as Universidades, o Conselho Federal de Medicina e o Conselho Brasileiro de Oftalmologia. Tem-se debatido sobre assuntos relevantes como a atualização do currículo médico, as deficiências na formação clínica geral dos médicos e a necessidade de revalidação do diploma médico e/ou dos títulos de especialidades ${ }^{(10-15)}$.

Este estudo mostrou dados positivos sobre as condições de realização deste exame na amostra em questão. Por exemplo, em nenhum caso o mesmo era realizado sem um esquema de suporte médico complementar para complicações decorrentes de reações adversas, seja um clínico/anestesista presente na sala, seja um clínico/anestesista de sobreaviso presente na clínica. Outro dado importante é que todas as clínicas estavam aparelhadas em relação à estrutura básica de equipamentos e medicações para o atendimento inicial de reações adversas ao contraste. 
Este estudo apresenta a limitação de retratar a realidade de uma região do Brasil, entretanto a maioria absoluta dos médicos entrevistados $(88,2 \%)$ possui o título do CBO, o qual somente é obtido através de prova de habilitação de abrangência nacional. E o $\mathrm{CBO}$, além de importante membro do International Council of Ophthalmology, é o maior órgão de classe oficial representante dos oftalmologistas em toda América Latina.

Os autores acreditam que é necessário um melhor treinamento na condução de reações adversas, durante o período de residência médica, principalmente para os oftalmologistas que irão executar este exame sem a presença de um anestesiologista, além de uma maior atenção às ações de educação médica continuada, a fim de manter atualizados os médicos com mais anos de conclusão do curso de especialização em oftalmologia. Serão necessários estudos posteriores mais detalhados a fim de ratificar estas observações e determinar melhor quais são as deficiências e como contorná-las.

\section{CONCLUSÕES}

Apesar das clínicas avaliadas contarem com boa estrutura física e disponibilidade de assistência por anestesista, os resultados deste estudo sugerem que a formação dos médicos oftalmologistas que realizam o exame de retinografia fluoresceínica apresenta deficiências em relação à condução de reações adversas deste contraste injetável, principalmente em médicos com mais de cinco anos de conclusão do curso de especialização.

\section{ABSTRACT}

Purposes: To evaluate the emergency resources of the clinics that perform fluorescein angiography in Pernambuco and, through a short multiple-choice questionnaire, to evaluate the ophthalmologists' performance to treat adverse reactions to contrast injection and to determine if the time after completing the specialization course in Ophthalmology influences in the results. Methods: An interview with the eighteen ophthalmologists of the ten clinics where this examination is performed was made. The multiple-choice questionnaire had ten questions with five options each and only one correct answer. This questionnaire was about adverse reactions to fluorescein injection. Results: One physician refused to participate and was excluded from the analysis. Among the others, fifteen physicians $(88.2 \%)$ were certified specialists by the Brazilian Council of Ophthalmologists. All clinics had the basic structure for the initial care of adverse reactions to contrast injection. Half of these clinics (five) performed this examination with the presence of an anesthesiologist directly monitoring the patient and the other half had one on duty, if necessary. The number of correct answers varied between 3 and 8, with a mean of 5.2 \pm 1.6 . Only four (23.6\%) physicians gave more than seven correct answers. Specialists with five or less years of postgraduation in Ophthalmology had better results regarding the questionnaire $(p<0.001)$. Conclusions: Despite appropriate material resources and support by anesthesiologist, the results of this study suggest that ophthalmologists who perform fluorescein angiography are not well-prepared to manage the possible adverse reactions, mainly doctors with more than five years after postgraduation in Ophthalmology.

Keywords: Fuorescein angiography; Fluorescein/adverse effect; Nausea/chemically induced; Vomiting/chemically induced; Contrast media; Anaphylaxis; Specialties, medical/ education; Questionnaires

\section{REFERÊNCIAS}

1. Berkow JW, Flower RW, Orth DH, Kelley JS. Fluorescein and indocyanine green angiography: techniques and interpretation. 2nd ed. San Francisco: The Foundation of the American Academy of Ophthalmology; 1997.

2. Helal Júnior J. Riscos da angiografia fluoresceínica. Arq Bras Oftalmol. 1981;44(2):99-100.

3. Karhunen U, Raitta C, Kala R. Adverse reactions to fluorescein angiography. Acta Ophthalmol (Copenh). 1986;64(3):282-6.

4. Kwiterovich KA, Maguire MG, Murphy RP, Schachat AP, Bressler NM, Bressler SB, et al. Frequency of adverse systemic reactions after fluorescein angiography: results of a prospective study. Results of a prospective study. Ophthalmology. 1991;98(7):1139-42.

5. Lacava AC, Leal EB, Caballero JC, Medeiros OA. Angiografia fluoresceínica e suas complicações: relato de um caso de óbito. Rev Bras Oftalmol. 1996; 55(1):59-62.

6. Yannuzzi LA, Rohrer KT, Tindel LJ, Sobel RS, Costanza MA, Shields W, et al. Fluorescein angiography complication survey. Ophthalmology. 1986; 93(5):611-7.

7. Conselho Brasileiro de Oftalmologia. Censo 2001. São Paulo: CBO; 2001. p.30.

8. Vilela MAP, Tock J, Abujamra S. Angiografia fluoresceínica. In: Abujamra S, Ávila M, Barsante C. Retina e vítreo: clínica e cirúrgica. São Paulo: Roca; 2000. p.91.

9. Brasil. Ministério da Saúde do Brasil. Agência Nacional de Vigilância Sanitária. Resolução RDC № 50, de 21 de fevereiro de 2002. Dispõe sobre o Regulamento Técnico para planejamento, programação, elaboração e avaliação de projetos físicos de estabelecimentos assistenciais de saúde. [citada 2005 mar 5]. Disponível em: http://www.cvs.saude.sp.gov.br/02rdc50.pdf

10. United States of America. American Academy of Ophthalmology. Continuing medical education (CME) Central. It is dedicated to offering ophthalmic education via Internet. [citada 2005 mar 5]. Disponível em: http://www.aao.org/ education/cme

11. Campos GWS. Educação médica, hospitais universitários e o Sistema Único de Saúde. Cad Saúde Pública. 1999;15(1)187-93.

12. Brasil. Conselho Brasileiro de Oftalmologia. Programa de Educação Médica Continuada. Dispõe sobre o projeto de educação à distância do $\mathrm{CBO}$ via $\mathrm{CD}$ ROM. [citada 2005 Mar 5]. Disponível em: http://www.cbo.com.br/cbo/ edcontinuada/index.htm

13. Brasil. Conselho Federal de Medicina. Educação Médica Continuada. Disponibiliza vídeos e textos sobre temas como Bioética, Medicina Brasileira e Práticas Médicas. [citada 2005 Mar 5]. Disponível em: http://www.portalmedico.org.br/ educacao_medica/emc.asp?portal

14. Ministério da Saúde do Brasil. Uma nova escola médica para um novo sistema de saúde. Saúde e Educação lançam programa para mudar o currículo de medicina. Rev Saúde Pública. 2002;36(3)375-8.

15. JAMA patient page. Your doctor education. JAMA. 2000;284(9):1198. 